\title{
On the Relationship of Tornado Path Length and Width to Intensity
}

\author{
HAROLD E. BROOKS \\ NOAA/National Severe Storms Laboratory, Norman, Oklahoma
}

(Manuscript received 14 January 2003, in final form 17 July 2003)

\begin{abstract}
Reported path lengths and widths of tornadoes have been modeled using Weibull distributions for different Fujita (F) scale values. The fits are good over a wide range of lengths and widths. Path length and width tend to increase with increasing F scale, although the temporal nonstationarity of the data for some parts of the data (such as width of F3 tornadoes) is large enough that caution must be exercised in interpretation of short periods of record. The statistical distributions also demonstrate that, as the length or width increases, the most likely F-scale value associated with the length or width tends to increase. Nevertheless, even for long or wide tornadoes, there is a significant probability of a range of possible $\mathrm{F}$ values, so that simple observation of the length or width is insufficient to make an accurate estimate of the $\mathrm{F}$ scale.
\end{abstract}

\section{Introduction}

The relationship of the size of tornadoes to the intensity of their damage is of importance in modeling the hazards associated with tornadoes (Schaefer et al. 1986, 2002; Meyer et al. 2002) and potentially could be useful in attempting to forecast intensity, if the relationships are found to be strong enough. Fujita and Pearson (1973), Tecson et al. (1979), and Abbey and Fujita (1975) described early efforts to look at the width and length of reported tornadoes in association with intensity, as measured by the Fujita scale, or F scale (Fujita 1981). McCarthy (2003) recently updated that work and showed that the width of tornado paths tends to increase with the intensity, but there is considerable overlap between classes.

If the distributions of width and length can be modeled with simple theoretical probability distributions, then the information associated with the thousands of observed tornado path lengths and widths could be summarized in two or three numbers. This offers obvious computational ease in comparison to tables based on empirical distributions, such as would produce histograms, as well as produces continuous functions, which seem physically more plausible. Development of statistically based hazard models for tornadoes could then use the parameters of the distributions as input. Changes in the parameterized distributions with time or space and differences between different classifications could also be investigated.

Herein I will describe an attempt to model the dis-

Corresponding author address: Harold E. Brooks, NOAA/NSSL, 1313 Halley Circle, Norman, OK 73069.

E-mail: Harold.Brooks@noaa.gov tribution of path lengths and widths using Weibull distributions. The relationship of path length and width to damage classification will be investigated, and an estimate of the probability of a particular F scale, given the length or width of a tornado, will be developed. Finally, the temporal robustness of the estimates and implications for interpretation of the data will be discussed.

\section{Data and methodology}

The dataset consists of all tornadoes in the National Weather Service (NWS) Storm Prediction Center (SPC) database of tornadoes in the United States from 1950-2001. Schaefer and Edwards (1999) and McCarthy (2003) described the database and the changes that have occurred over the years, including the effects of increasing verification efforts and public awareness. The database is available online from the SPC Web site (http://www.spc.noaa.gov). Path lengths are reported in miles, widths in yards, ${ }^{1}$ and the maximum damage as rated by the F scale. Doswell and Burgess (1988) have discussed problems with assigning F-scale ratings, but it is hoped that the relatively large sample size here will overcome random errors in assignments. If systematic biases exist, they are extremely difficult to detect and there is nothing that can be done about them. Only those events with a reported length or width are included in the analysis. Over 40000 tornadoes are included.

Weibull distributions have been fit to the observed

\footnotetext{
${ }^{1}$ The mean width was reported prior to and including 1994 and the maximum width after 1994.
} 
path length and width data. ${ }^{2}$ Weibull distributions are appropriate for the problem because they are nonnegative and have positive skewness. They have been used historically to model wind speed distributions (Wilks 1995). The probability distribution function for a Weibull distribution is given by

$$
f(x)=\left(\frac{\alpha}{\beta}\right)\left(\frac{x}{\beta}\right)^{\alpha-1} \exp \left[-\left(\frac{x}{\beta}\right)^{\alpha}\right],
$$

where $\alpha$ and $\beta$ are the two parameters (shape and scale, respectively) that define the distribution, and $x, \alpha$, and $\beta$ are greater than zero. If $\alpha \leq 1$, the distribution is shaped like a reverse "J," with maximum values of the function as $x \rightarrow 0$. For $\alpha>1$, the function peaks at greater value of $x$. If $\alpha=1$, the distribution reduces to the exponential distribution. The scale parameter, $\beta$, stretches or compresses the distribution along the $x$ axis for any particular value of $\alpha$. The parameters have been estimated with a maximum likelihood technique by using software included in the U.S. Department of Defense's (2002) Composite Materials Handbook. The mean of the Weibull distribution, $\mu$, is given by

$$
\mu=\beta \Gamma\left(1+\frac{1}{\alpha}\right),
$$

where $\Gamma()$ is the gamma function. In this work, the argument of the $\Gamma()$ ranges from approximately 0.6 to 1.1 for most calculations. As a result, typically, $0.9 \beta<$ $\mu<1.5 \beta$, so that the mean width and length are on the order of $\beta$.

The distributions have been fit over a variety of time intervals. Sample-size limitations make interpretation of some of those time intervals for the highest values of the F scale difficult. For example, there are only 51 F5 ${ }^{3}$ tornadoes in the record, so that interpretation of the distributions on anything less than the complete record for F5 tornadoes is questionable. On the other hand, there are approximately $7500 \mathrm{~F} 2$ tornadoes with path length and width information, so that short time ranges provide an adequate sample size.

\section{Results}

\section{a. The complete record}

Quantile-quantile $(q-q)$ plots are a useful tool to assess the goodness of fit of the models qualitatively (Wilks 1995). Quantile-quantile plots compare the values associated with the same quantile of an empirical (observed) and modeled probability distribution function. For example, a plot of the 10th, 20th, 30th, etc.,

\footnotetext{
${ }^{2}$ Lognormal and Gumbel distributions (see Wilks 1995) have also been fit to the observed data but were clearly much poorer fits, so they are not shown.

3 "Fn" implies that the tornado was rated as " $\mathrm{n}$ " on the F scale, regardless of its true meteorological intensity.
}
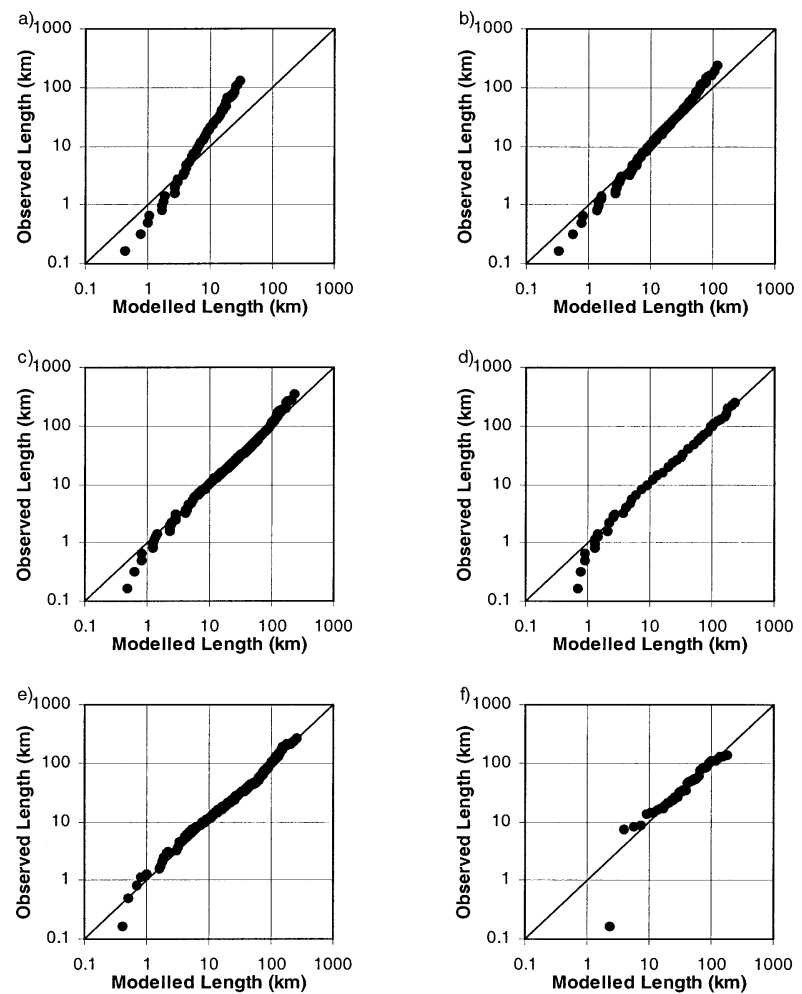

FIG. 1. Quantile-quantile plots for Weibull distribution fits to path length data for tornadoes of F scales (a) F0 to (f) F5 from 1950 to 2001. Note logarithmic axes. Diagonal line indicates perfect fit.

percentiles of the empirical distribution versus the 10th, 20th, 30th, etc., percentiles of the modeled distribution shows how well the data are fit by the model. In this case, the quantiles are plotted for all values at which the empirical quantiles exist. The "quantization" of the reports is a limit of how these plots can be made. As an example, the F1-length $q-q$ plot begins with the $22 \mathrm{~d}$ percentile, since $22 \%$ of the observations have path lengths of 0.1 mile $(0.16 \mathrm{~km})$. The gaps in the plots are a result of gaps in the observational record.

The fits to the length data improve with increasing $\mathrm{F}$ scale. The fits are not especially good at F0 or, to a lesser extent, F1, although they are better than fits assuming a normal distribution for all values. In general, all of the modeled distributions overestimate the empirical distribution for short lengths, as indicated by the points being below the diagonal, perfect-fit line (Fig. 1). The problems at short lengths are a result of the quantization of the observations. The F0 tornado fit departs from the observations at about $4 \mathrm{~km}$, underestimating the distribution for long lengths (Fig. 1a), but it is important to note that this is at the $93 \mathrm{~d}$ percentile, so that the errors are for rare events. The underestimation of density at long path lengths decreases for F1 and F2 tornadoes and is very small by F3 tornadoes. The picture is very similar for the widths (Fig. 2). Fits improve with 

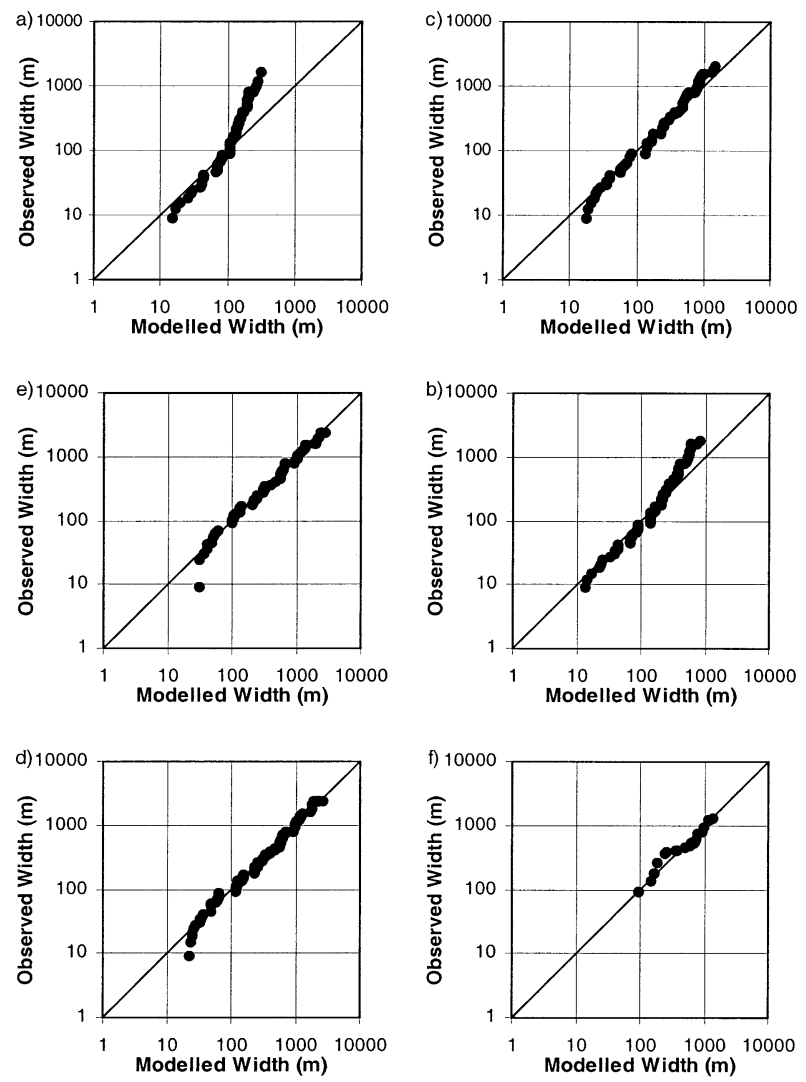

FIG. 2. Same as Fig. 1, except for path width.
TABLE 1. Parameters of the Weibull distributions for fits to length data for 1950-2001, where $\mathrm{F}$ is the $\mathrm{F}$ scale, $N$ is the number of tornadoes in each class, $\alpha$ and $\beta$ are the parameters of the distribution, and $\mu$ is the mean of the distribution; $\beta$ and $\mu$ are in $\mathrm{km}$.

\begin{tabular}{crrrr}
\hline \hline & \multicolumn{4}{c}{ Weibull parameter } \\
\cline { 2 - 5 } F scale & \multicolumn{1}{c}{$\alpha$} & $\alpha$ & \multicolumn{1}{c}{$\beta$} & \multicolumn{1}{c}{$\mu$} \\
\hline 0 & 16441 & 0.65 & 0.9 & 1.4 \\
1 & 14450 & 0.62 & 3.1 & 4.7 \\
2 & 7503 & 0.64 & 7.5 & 10.7 \\
3 & 1967 & 0.83 & 20.4 & 22.5 \\
4 & 469 & 1.01 & 43.9 & 43.6 \\
5 & 51 & 1.20 & 57.7 & 54.6 \\
\hline
\end{tabular}

increasing width, and the statistical distribution tends to overestimate the density at low widths.

The calculated mean path length for tornadoes increases from a little over $1 \mathrm{~km}$ for F0 tornadoes to over $50 \mathrm{~km}$ for F5 tornadoes (Table 1). The mean length roughly doubles with each value of the $\mathrm{F}$ scale from $\mathrm{F} 1$ to F4. The change from F0 to F1 is somewhat larger, and there is less change from F4 to F5. The shape of the distribution changes as well, with the $\alpha$ parameter increasing, in general, with increasing $\mathrm{F}$ scale. This implies that the maximum probability moves away from zero length for the high $\mathrm{F}$ values. The distributions for the different F-scale values are well separated except for perhaps between F4 and F5, as illustrated by the cumulative distribution functions for the various values of F (Fig. 3.)

The mean width also increases with $\mathrm{F}$ scale (Table

\section{Cumulative Distribution Functions for Path Length}

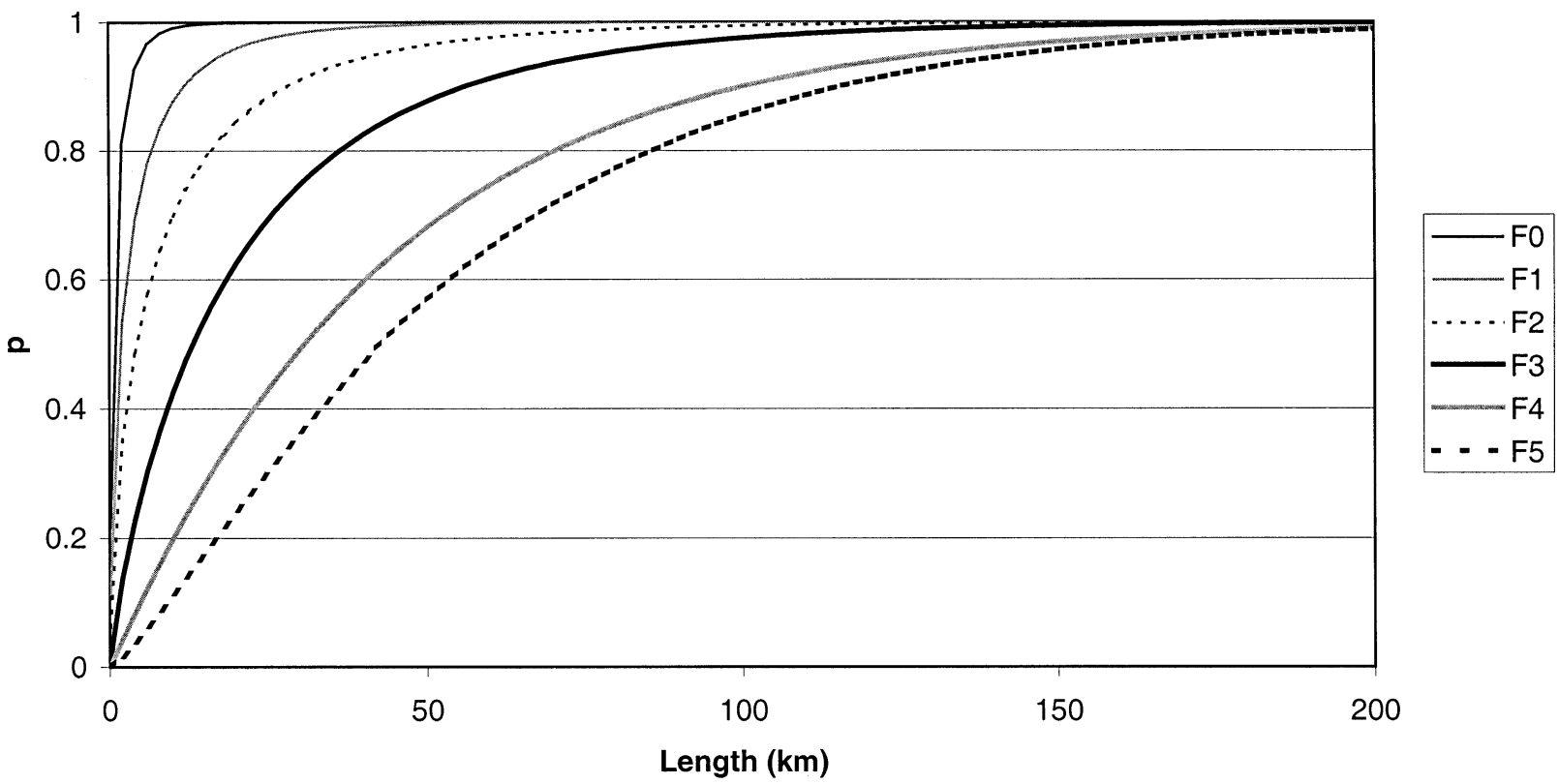

FIG. 3. Cumulative distribution functions for path length of tornadoes by F scale. Thin lines are F0-F2 and thick lines are F3-F5, with solid, gray, and dashed lines representing the three F-scale values in each group, respectively. 
TABLE 2. Same as Table 1, except for width data in $\mathrm{m}$.

\begin{tabular}{crrrr}
\hline \hline & \multicolumn{4}{c}{ Weibull parameter } \\
\cline { 2 - 5 } F scale & \multicolumn{1}{c}{$N$} & \multicolumn{1}{c}{$\alpha$} & \multicolumn{1}{c}{$\beta$} & \multicolumn{1}{c}{$\mu$} \\
\hline 0 & 16166 & 0.94 & 27.2 & 28.4 \\
1 & 14383 & 0.85 & 57.7 & 64.0 \\
2 & 7487 & 0.79 & 107.4 & 125.9 \\
3 & 1960 & 0.84 & 240.2 & 263.6 \\
4 & 469 & 1.00 & 461.2 & 460.7 \\
5 & 51 & 1.71 & 620.5 & 555.5 \\
\hline
\end{tabular}

2), from less than $30 \mathrm{~m}$ for $\mathrm{F} 0$ to more than $550 \mathrm{~m}$ for F5. It doubles approximately with each $\mathrm{F}$ value from F0 to F4, with only a slight increase from F4 to F5. There is less consistency in the change in the $\alpha$ parameter than in the case of path length. Only the F5 tornadoes have $\alpha$ greater than 1. The distributions are less distinct for the high $\mathrm{F}$ values, particularly at wide widths (Fig. 4).

The distributions represent the probability of a path length (width) given that a tornado of a particular intensity occurs. It is easy to invert the problem to calculate the probability of a particular intensity given that a path length (width) is observed. To do so, all that must be done is to weight the distributions given by (1) by the number of tornadoes at each $\mathrm{F}$ scale and then divide the result for each F scale by the sum of the weighted distributions at any given length (width). The results illustrate the potential utility and pitfalls of estimating a damage rating simply from the length or width data associated with a tornado.

At short path lengths $(<5 \mathrm{~km})$, the most probable $\mathrm{F}$ scale is F0 (Fig. 5). Over a broad range ( $\sim 30-170 \mathrm{~km})$, the most likely F scale is F2. Over that range, however, there is a significant probability of at least 5\% for any value between F1 and F4. Length information appears to be potentially of some limited utility for setting lower bounds on the likely intensity of tornadoes. Less than $5 \%$ of tornadoes with path lengths of $25 \mathrm{~km}$ are rated F0. The 5\% cutoff for all F0 or F1 tornadoes is approximately $160 \mathrm{~km}$. Given the rarity of tornadoes of that length (the most recent one in the record was in 1992), this is not a strong result in a practical sense. It is also of interest to note that, because of the rarity of F5 tornadoes, they are never the most likely event at any length.

Width information potentially has more value in putting limits on the likely intensity rating (Fig. 6). Less than $20 \%$ of tornadoes with widths of $400 \mathrm{~m}$ and less than $10 \%$ of tornadoes with widths of $500 \mathrm{~m}$ are rated F0 or F1. Thus, one can have some confidence that a wide tornado is probably at least F2. Finer distinctions based on width are difficult to justify. From 500 to 1500 $\mathrm{m}$, the distributions suggest that there is at least a $10 \%$ probability that a tornado will be F2, F3, or F4. Again, because of the rarity of F5 tornadoes, they are never the most likely event at any width.

\section{b. 4-yr periods}

Consideration of shorter time periods in the record allows for the opportunity to look at the stability of the estimates of the parameters in time and to put confidence limits on the long-term values. The typical number of

\section{Cumulative Distribution Function for Path Width}

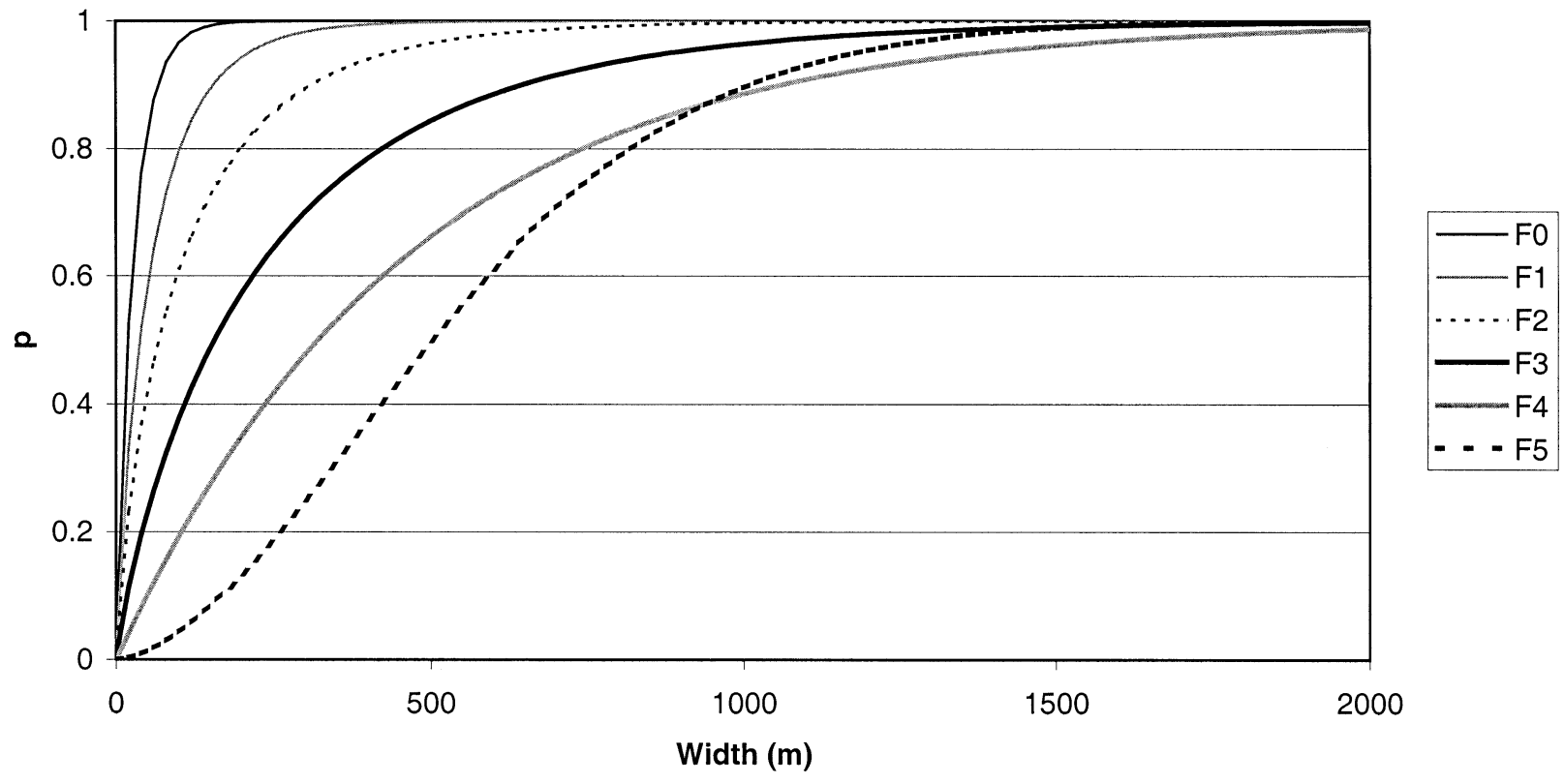

FIG. 4. Same as Fig. 3 except for path width. 


\section{Probability of F-scale Given Length}

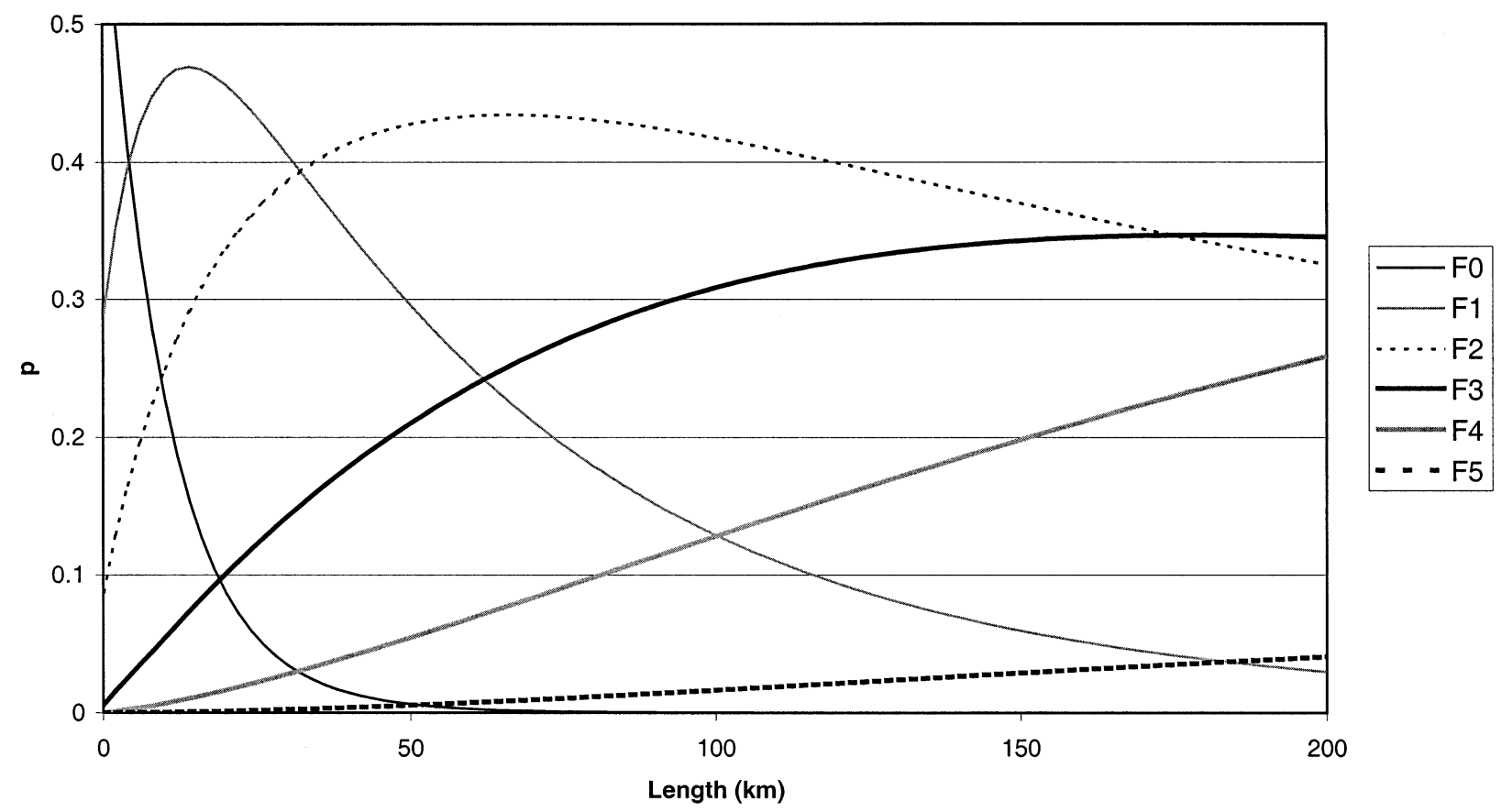

FIG. 5. Probability $(p)$ of F-scale value, given path length. Convention for lines as in Fig. 3.

Probability of F-scale Given Width

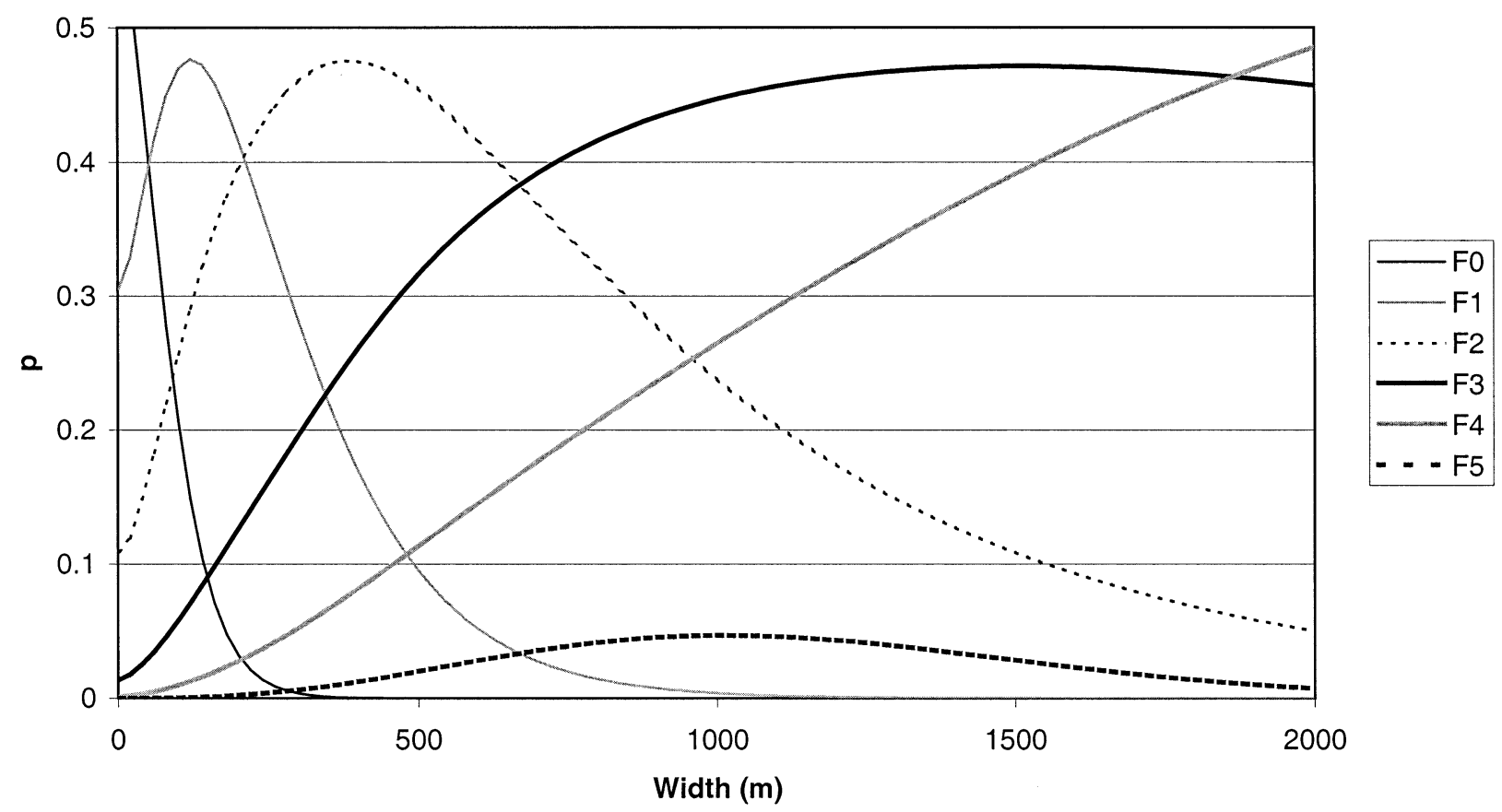

FIG. 6. Same as Fig. 5, except for path width. 

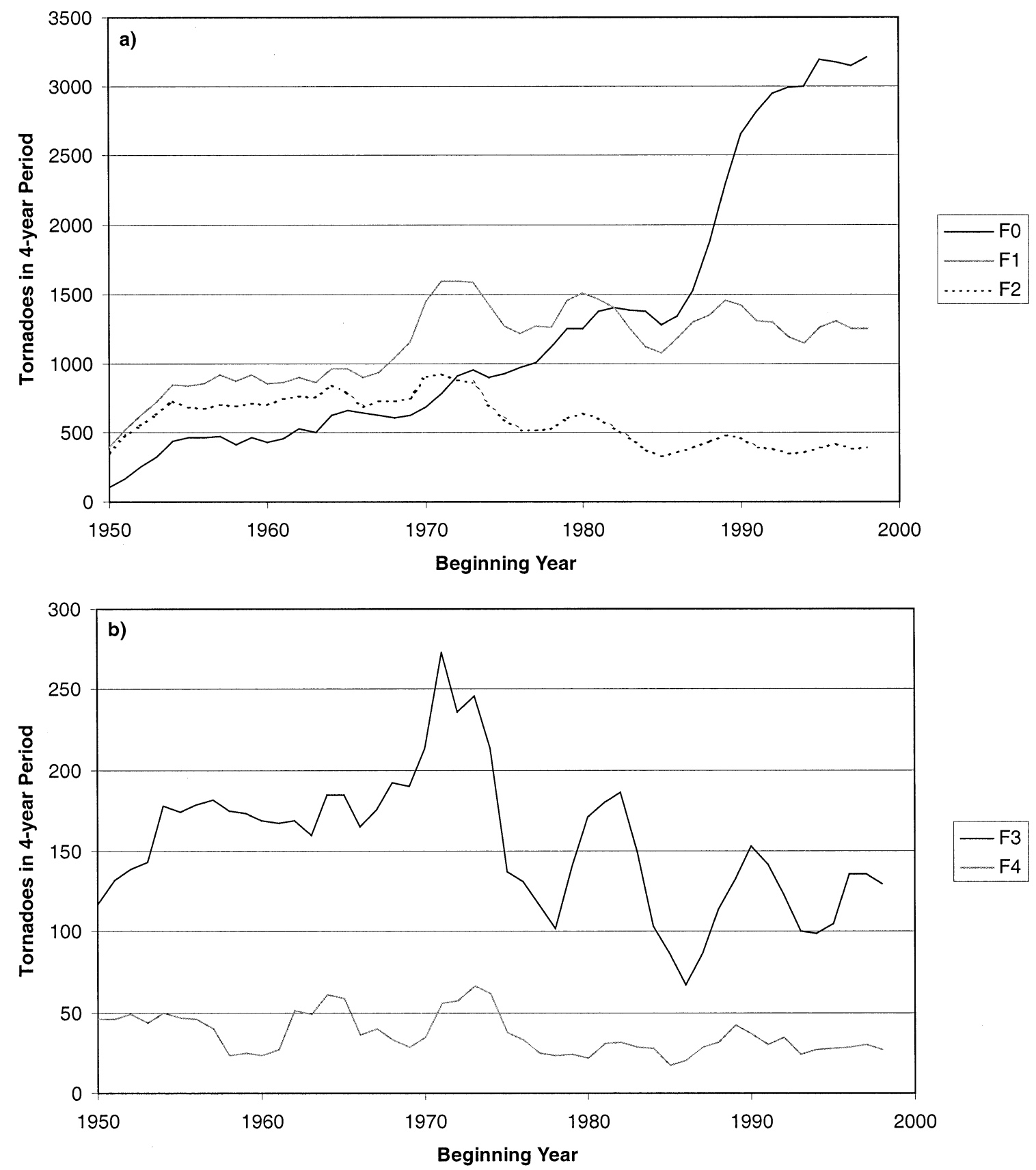

FIG. 7. Number of tornadoes in overlapping 4-yr periods by F scale: (a) F0 (black solid), F1 (gray solid), and F2 (dashed); (b) F3 (black solid) and F4 (gray solid).

reports per year varies dramatically through time, as illustrated by the number of reports in overlapping 4yr periods (Fig. 7) ${ }^{4}$ The rapid increase at the beginning of the period of record is likely a result of the beginning of real-time forecasting of tornadoes by the NWS, with

${ }^{4} \mathrm{~F} 5$ tornado reports are not shown because of their small number. There are no more than 11 in any 4 -yr period, and in most periods there are no more than 5. Clearly, estimates of the statistical distribution, based on such a small sample, would have little meaning. the beginning of the Severe Weather Unit of the Weather Bureau in 1952 through the move of the unit from Washington, D.C., to Kansas City, Missouri, in 1954 (Corfidi 1999). The number of weak tornadoes (F0 and F1) has increased dramatically in the dataset, with the F0 increasing particularly when data from the year 1990 are first included. Part of this increase for F0 tornadoes is a result of a policy change in 1982 to assign F0 to all tornadoes that did not have rated damage (D. McCarthy, 2003 personal communication). An additional compo- 
Distribution of Mean Path Length Estimates

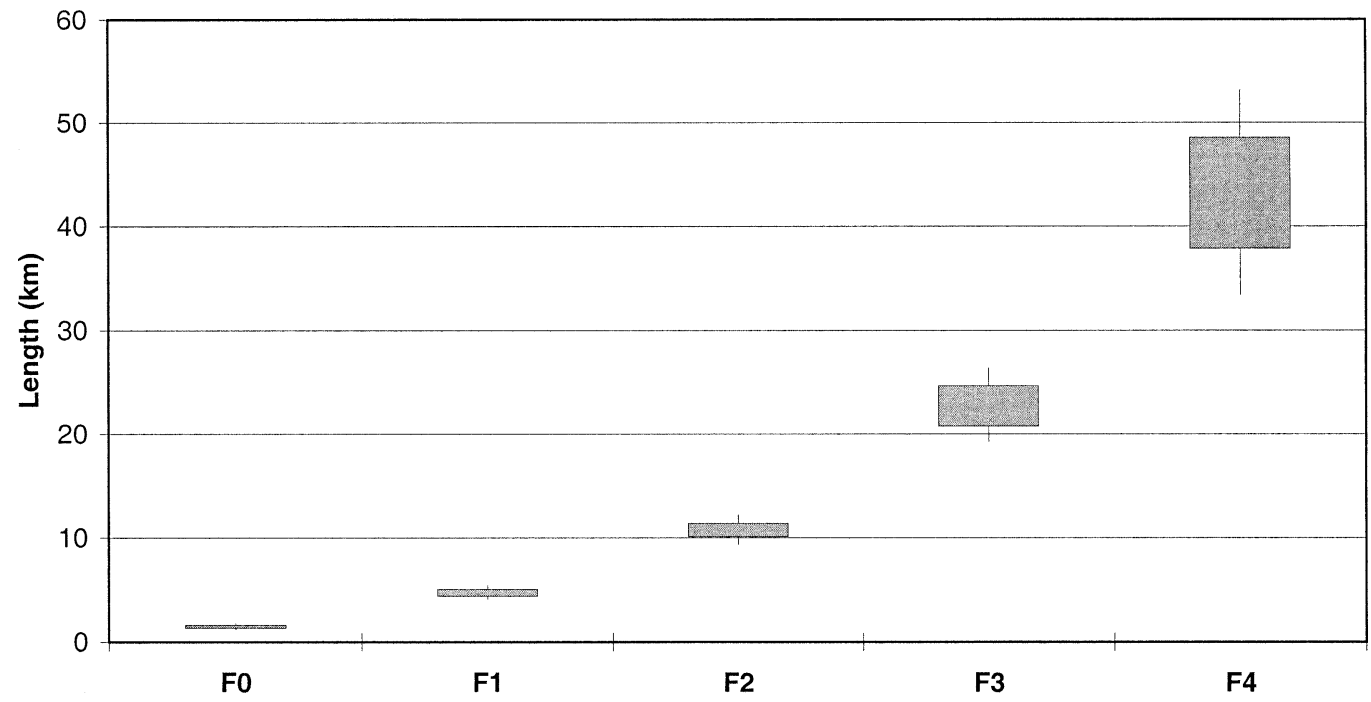

FIG. 8. Box-and-whisker plots for distribution of mean path length estimates based on random samples of 4 yr Tops and bottoms of boxes represent 75 th and 25 th percentiles, respectively. Tops and bottoms of whiskers represent 90th and 10th percentiles, respectively.

nent of the increase is likely due to increasing population in the western United States and better public awareness. The numbers of $\mathrm{F} 2$ and stronger tornadoes show a slightly downward trend, with an apparent step function decrease in the mid-1970s. The step function may be a result of the beginning of real-time damage surveys when the Fujita scale was adopted by the NWS. Almost all 4-yr periods have at least $100 \mathrm{~F} 3$ tornadoes and 20 F4 tornadoes in them. Thus, the sample size allows some hope of estimating the parameters of the distribution from a 4-yr period.
In order to get a handle on variability, I have randomly selected 4 yr out of the record, calculated the parameters from those years, and then repeated that process 1000 times to compute distributions of the parameters. Box and whisker plots illustrate the variability of the mean values (Figs. 8 and 9). Random collections of years clearly show a distinction between the different $F$ values, with more damaging tornadoes being longer and wider.

The estimates are not consistently robust in time, however. Most, such as the mean length of F3 tornadoes,

Distribution of Mean Path Width Estimates

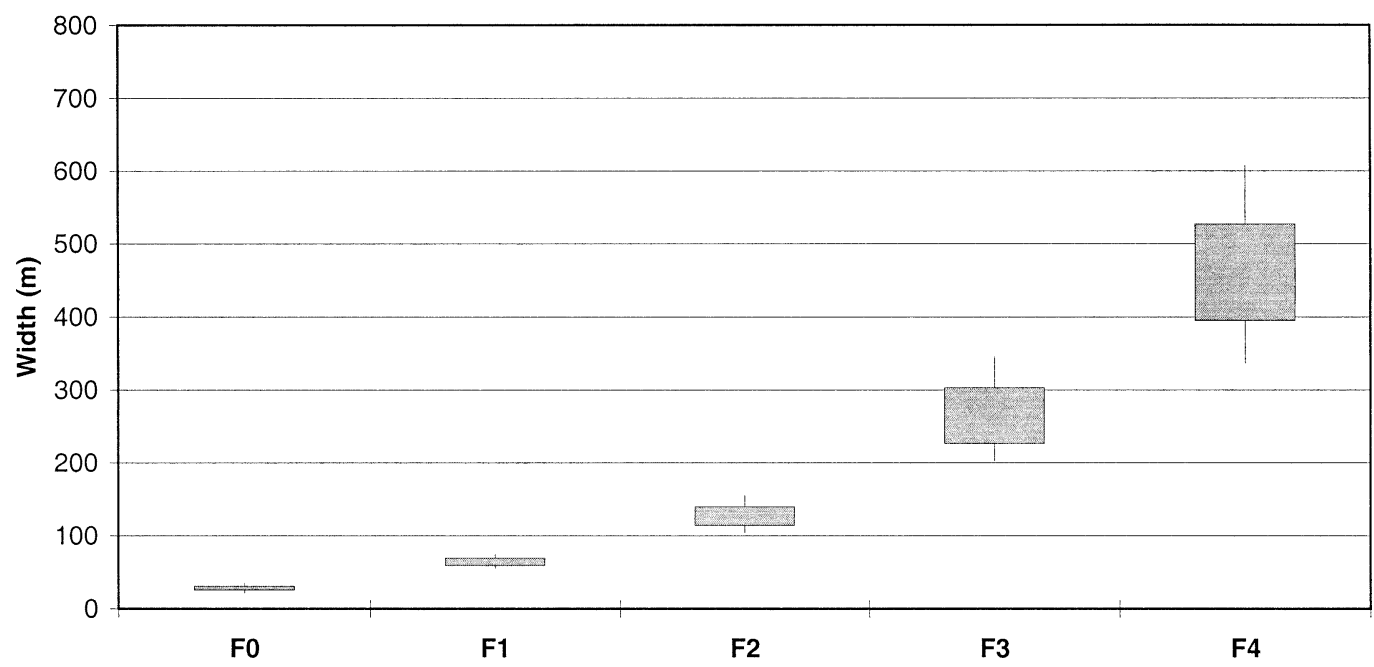

FIG. 9. Same as Fig. 8, except for path width estimates. 


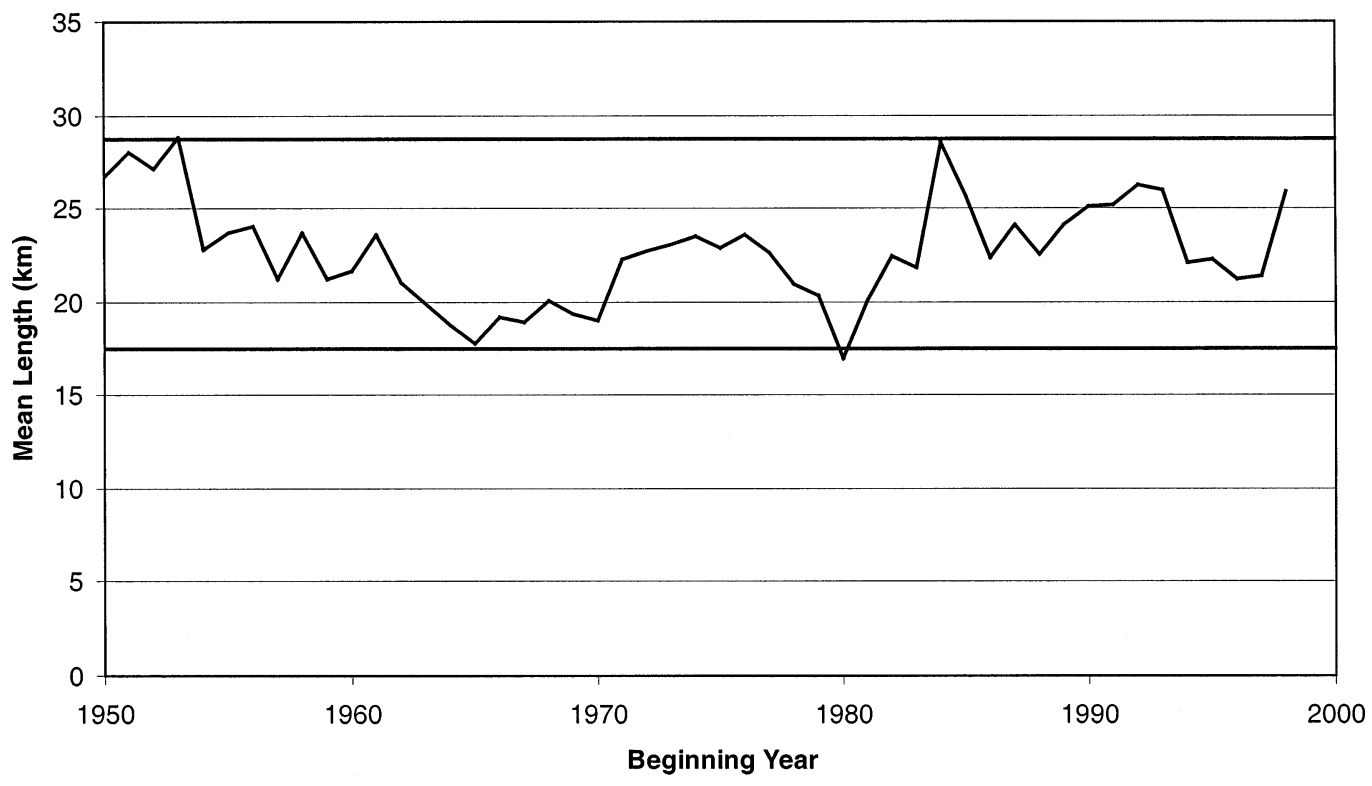

FIG. 10. Mean length of distribution of F3 tornadoes for overlapping 4-yr periods. Bold horizontal lines represent $95 \%$ confidence intervals on long-term mean.

are quite consistent in time (Fig. 10). Others, particularly associated with the weaker tornadoes, show a decrease in the early periods, as illustrated by the mean width of F0 tornadoes (Fig. 11). This could be explained by the greater ease of detecting the effects of only the largest of all weak tornadoes. Detection efficiency of weak tornadoes is always a problem in climatological studies, and it may be that the larger ones are more likely to be detected. As a result, in the period of poor detection, only the largest weak tornadoes make the record, biasing the results upward.
Perhaps the most puzzling record is the widths of F3 tornadoes, which shows a slow increase beginning in early 1970s, when the mean reported path width was a little less than $200 \mathrm{~m}$, to the mid-1990s, when the mean reported width was over $500 \mathrm{~m}$, followed by a rapid decrease in the late 1990s and early 2000s (Fig. 12). Both the minimum and the maximum 4-yr periods are outside of the $95 \%$ confidence intervals for the longterm mean. The peak estimate for the width is higher than any of the random combinations that went into the estimates shown in Fig. 9, which peaked at $467 \mathrm{~m}$. It

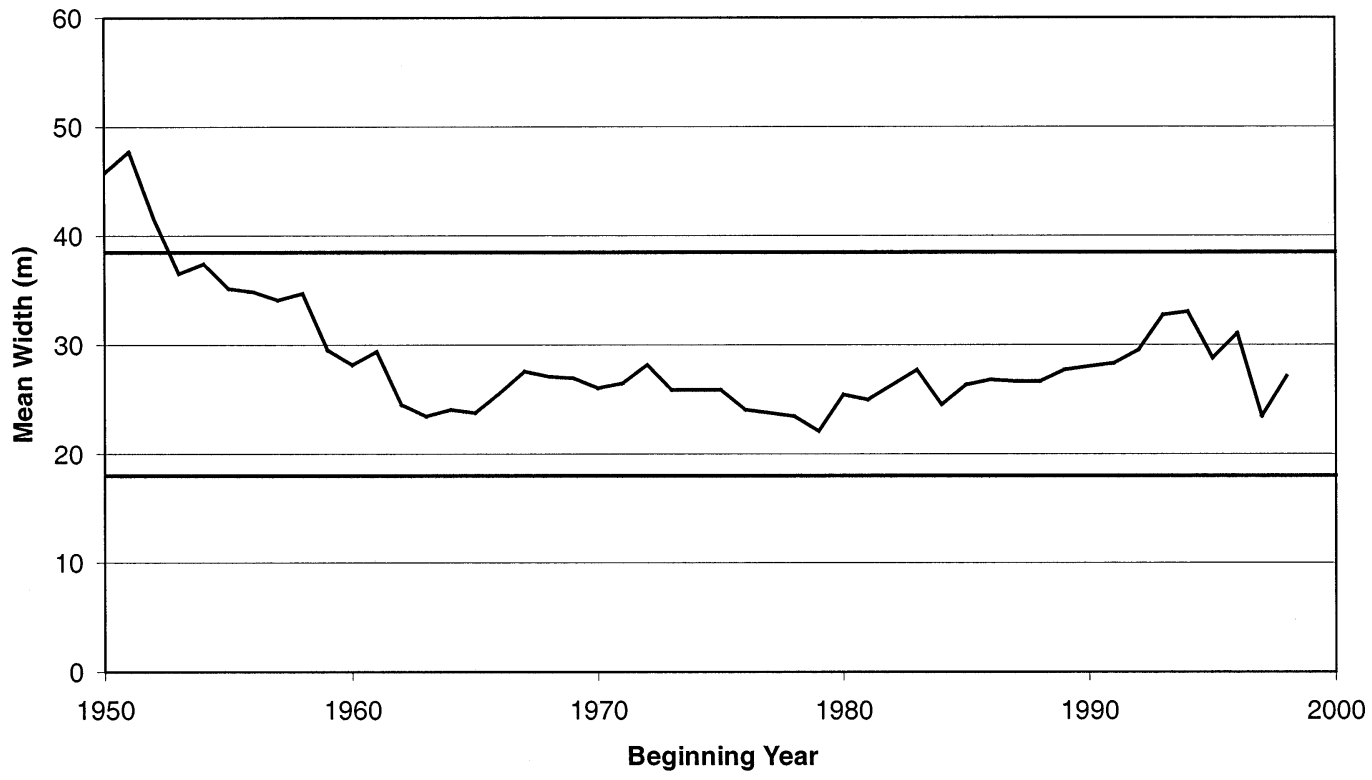

FIG. 11. Same as Fig. 10 except for mean width of F0 tornadoes. 


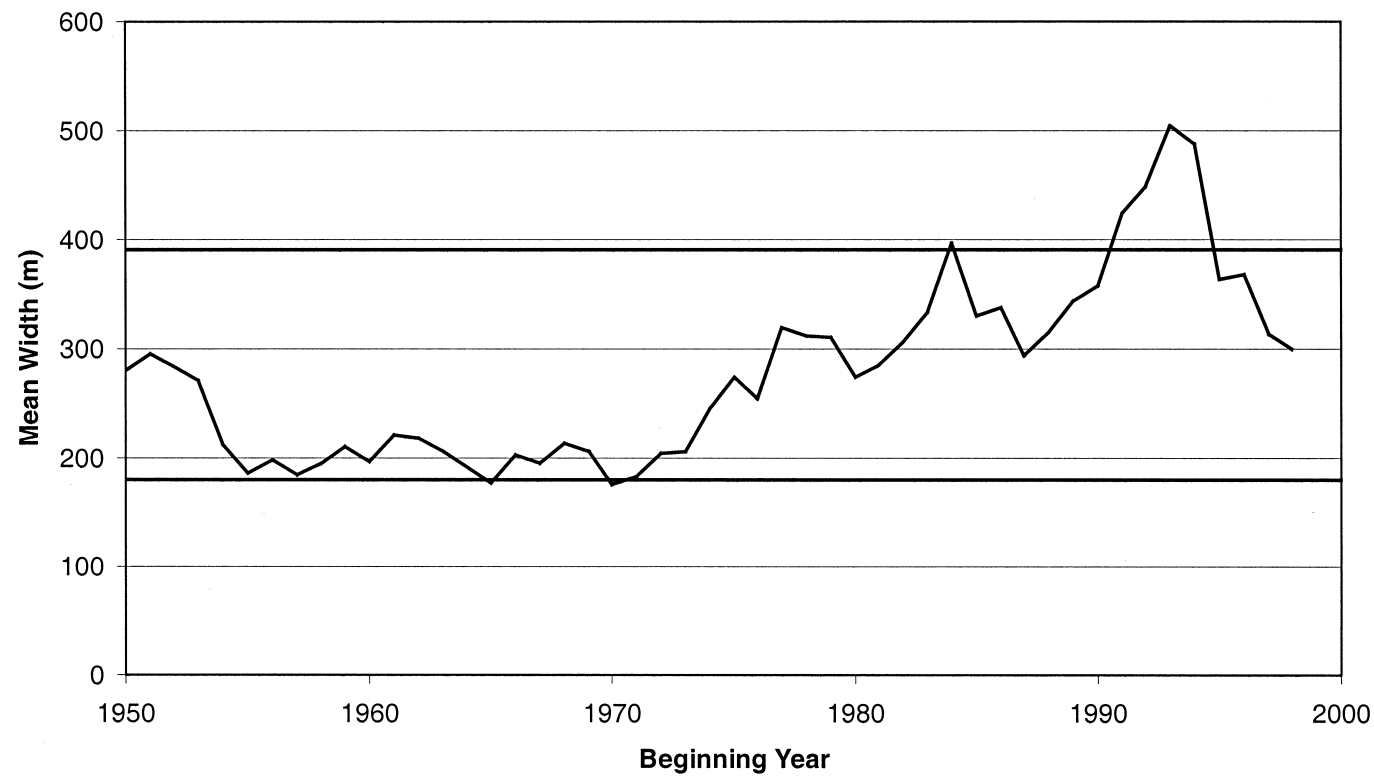

FIG. 12. Same as Fig. 10 except for mean width of F3 tornadoes.

fits well in the overall distribution of widths for F4 tornadoes, so that mid-1990s F3 tornadoes had reported widths comparable to the long-term record of F4 tornadoes. Although there was a policy change in the NWS to report maximum path width, instead of mean path width, that did not occur until 1994 (McCarthy 2003). Thus, the width increase takes place for a long period of time before the policy change, and a decrease follows not long after the policy change. It is not easy to explain why the mean width would increase so much. Maximum width might be expected to increase with greater concentration on damage surveys, if a greater fraction of the path is surveyed. It is not obvious that the effect on the mean path should be very large, and it is not obvious at all why it should occur with the F3 tornadoes and not with others. It may be nothing more than coincidence, but it suggests that caution should be applied to statistics from short periods of record.

\section{Concluding remarks}

Weibull distributions provide reasonably good fits to the record of reported tornado path lengths and widths. The goodness of the fits implies that Weibull distributions may be useful in developing statistically based models of tornado hazards, especially given the fact that a closed form exists for the cumulative distribution function, making computations with them very efficient if a large number of tornadoes are being simulated. For any comparable period of record, lengths and widths in general increase with increasing damage scale. Physically, this seems plausible since a longer path length means that there is more opportunity for damage to occur and, in general, longer path lengths are associated with longer-lived tornadoes and/or faster storm motions.
Assuming that the tornado is nearly symmetric, wider tornadoes will take longer to pass over a particular point, giving more time for damage to occur even if peak wind speeds are the same. There is sufficient nonstationarity in the time series, however, that comparison of different periods of record could lead to confusion, so that caution must be exercised in producing parameters. As an example, F3 tornado widths are far from stationary. The timing of changes in reported parameters such as the path width do not match up particularly well with the timing of policy changes, leaving open the question of the true effects of those policy changes. It is possible that subtle "unofficial" changes in the way that damage path information is collected might have an impact on the statistics.

From inverting the probability distributions for size versus $\mathrm{F}$ scale to give a probability of $\mathrm{F}$ scale given size, it is clear that, in general, the most likely F scale increases with increasing reported tornado size, although F5 tornadoes are never the most likely event for any size. It is also clear, though, that for most lengths and widths, there is a wide range of F-scale values associated with significant probability of occurrence. Since it is possible to get an estimate of the width in some cases from observing tornadoes in real time, it may be possible to guess that it is highly likely that a wide tornado (say, $500 \mathrm{~m}$ ) is of at least F2 intensity, but going much beyond that statement is questionable. Thus, the use of length and width in forecasting intensity is of limited value.

Acknowledgments. Thanks are expressed to Dan McCarthy and John Hart of the NWS Storm Prediction Center for providing the data analyzed. Mr. McCarthy also provided valuable insights into historical policy 
changes and a helpful review of a draft. Cathryn Meyer carried out an early version of the fitting to distributions.

\section{REFERENCES}

Abbey, R. F., and T. T. Fujita, 1975: Use of tornado path lengths and gradations of damage to assess tornado intensity probabilities. Preprints, Ninth Conf. on Severe Local Storms, Norman, OK, Amer. Meteor. Soc., 286-293.

Corfidi, S. F., 1999: The birth and early years of the Storm Prediction Center. Wea. Forecasting, 14, 507-525.

Doswell, C. A., III, and D. W. Burgess, 1988: On some issues of United States tornado climatology. Mon. Wea. Rev., 116, 495501.

Fujita, T. T., 1981: Tornadoes and downbursts in the context of generalized planetary scales. J. Atmos. Sci., 38, 1511-1534.

- , and A. D. Pearson, 1973: Results of FPP classification of 1971 and 1972 tornadoes. Preprints, Eighth Conf. on Severe Local Storms, Denver, CO, Amer. Meteor. Soc., 142-145.

McCarthy, D. W., 2003: NWS tornado surveys and the impact on the national tornado database. Preprints, First Symp. on F-Scale and Severe-Weather Damage Assessment, Long Beach, CA, Amer. Meteor. Soc., CD-ROM, 3.2.

Meyer, C. L., H. E. Brooks, and M. P. Kay, 2002: A hazard model for tornado occurrence in the United States. Preprints, 16th Conf. on Probability and Statistics, Orlando, FL, Amer. Meteor. Soc., J88-J95.

Schaefer, J. T., and R. Edwards, 1999: The SPC tornado/severe thunderstorm database. Preprints, 11th Conf. on Applied Climatology, Dallas, TX, Amer. Meteor. Soc., 215-220.

_ _ D. L. Kelly, and R. F. Abbey, 1986: A minimum assumption tornado-hazard probability model. J. Climate Appl. Meteor., 25, 1934-1945.

_ hazard estimates. Preprints, Third Symp. on Environmental Applications, Orlando, FL, Amer. Meteor. Soc., 35-41.

Tecson, J. J., T. T. Fujita, and R. F. Abbey, 1979: Statistics of U.S. tornadoes based on the DAPPLE (Damage Area Per Path LEngth) tornado tape. Preprints, Eleventh Conf. on Severe Local Storms, Kansas City, MO, Amer. Meteor. Soc., 227-234.

U.S. Department of Defense, 2002: Composite materials handbook. Vol. 1. Polymer Matrix composites: Guidelines for characterization of structural materials. MIL-HDBK-17-1F, U.S. Dept. of Defense, 586 pp. [Available from Document Automation and Production Service, Bldg. 4D, DODSSP/ASSIST, 700 Robbins Ave., Philadelphia, PA 19111-5094 or online at http://snebulos.mit.edu/projects/reference/milstd/ MIL-HDBK-17-1.pdf.]

Wilks, D. S., 1995: Statistical Methods in the Atmospheric Sciences. Academic Press, 467 pp. 